\title{
EL CONTROL DE DATOS DE CIRCULACIÓN DE PERSONAS EN LA UE COMO MECANISMO DE SALVAGUARDA DE LA SEGURIDAD NACIONAL
}

ROSARIO SERRA CRISTÓBAL 
SUMARIO

1. EL TERRORISMO YIHADISTA COMO FENÓMENO TRANSFRONTERIZO. 2. LA IMPORTANCIA DEL INTERCAMBIO DE DATOS SOBRE EL TRASPASO DE FRONTERAS EN EL ESPACIO SCHENGEN. 3. LAS NUEVAS DIRECTIVAS SOBRE EL TRATAMIENTO DE DATOS Y LA SALVAGUARDA DE LA SEGURIDAD NACIONAL. 4. EL REGISTRO DE PASAJEROS (EL PERSONAL NAME RECORD O PNR). 5. CUANDO LOS DATOS CRUZAN LAS FRONTERAS EXTERNAS. EL INTERCAMBIO DE DATOS CON TERCEROS PAÍSES AJENOS A LA UE. 5.1. EL FALLIDO ACUERDO PNR CON CANADÁ Y LAS EXIGENCIAS DEL TRIBUNAL DE JUSTICIA EN MATERIA DE CESIÓN DE DATOS DE PASAJEROS. 5.2. EL INTERCAMBIO DE DATOS CON ESTADOS UNIDOS. EL EU-US UMBRELLA AGREEMENT Y EL PRIVACY SHIELD. 6. EL USO EN LAS FRONTERAS DE PERFILES Y LISTAS NEGRAS DE PRESUNTOS TERRORISTAS. 7. A MODO DE CONCLUSIÓN 


\title{
EL CONTROL DE DATOS DE CIRCULACIÓN DE PERSONAS EN LA UE COMO MECANISMO DE SALVAGUARDA DE LA SEGURIDAD NACIONAL ${ }^{1}$
}

\author{
ROSARIO SERRA CRISTÓBAL ${ }^{2}$ \\ Prof. Titular de Derecho Constitucional \\ Universidad de Valencia
}

\section{EL TERRORISMO YIHADISTA COMO FENÓMENO TRANSFRONTERIZO}

El terrorismo yihadista es un terrorismo con características nuevas ${ }^{3}$ : estructura dispersa, con alto grado de fanatismo que puede conducir a inmolaciones con un elevado poder de causar daños, búsqueda constante de nuevas vulnerabilidades y novedosos instrumentos de captación, adiestramiento o adoctrinamiento en el odio ${ }^{4}$.

1 Este trabajo se ha realizado en el marco del Proyecto de I+D «Seguridad Global y Derechos fundamentales: protección contra las amenazas y garantías de las libertades» (Referencia: DER201565288-R) financiado por el Ministerio de Economía, Industria y Competitividad y la Agencia Estatal de Investigación y FEDER.

2 Departamento de Derecho Constitucional. Universidad de Valencia. Av. Los Naranjos, s/n, Valencia 46022. Email: rosario.serra@uv.es

3 Es más, como indicaba González Cussac, «desde los atentados del 11 de septiembre, el nuevo terrorismo ya no se analiza como una cuestión meramente criminal o nacional, sino que comporta otras muchas proyecciones que el viejo terrorismo nunca tuvo». Es un terrorismo que no posee una regulación precisa, su objetivo estratégico es provocar el colapso de la retaguardia civil, y es un terrorismo que intenta aprovechar las potencialidades del enemigo en beneficio propio, explota las libertades de las sociedades democráticas para cubrir sus actividades ilegales. GONZÁLEZ CUSSAC, José L. (2016). «Servicios de inteligencia y contraterrorismo». Guillermo Portilla Contreras y Ana Isabel Pérez Cepeda (Dirs.), Terrorismo y contraterrorismo en el Siglo XXI. Un análisis penal y político crimininal, Madrid, Ratio Legis, págs. 117 y 120.

4 DE VERGOTTINI (2004). «La difícil convivencia entre libertad y seguridad. Respuestas de las democracias al terrorismo». Revista de Derecho Político, n. 61, pág. 24. 
Como se ha señalado, la amenaza del terrorismo yihadista existe en Europa Occidental desde el inicio de la década de los noventa del siglo xx. Pero los procesos de radicalización y reclutamiento asociados con esa amenaza nunca antes han sido tan extensos e intensos en las sociedades europeas como lo son ahora ${ }^{5}$.

Estos grupos terroristas han demostrado tener amplias habilidades en el manejo de las redes telemáticas no solo para la financiación de sus actividades sino también para la captación y adoctrinamiento de adeptos a la causa yihadista ${ }^{6}$. Se caracteriza por su vocación de expansión internacional, a través de líderes carismáticos que difunden sus consignas por medio de internet y, especialmente, mediante el uso de redes sociales, realizando un llamamiento a seguidores de todo el mundo para que cometan atentados. Como consecuencia, es difícil su persecución, es un enemigo al que no siempre se le ve y que puede aparecer como célula aislada en el rincón de cualquier ciudad ${ }^{7}$. Como se ha subrayado, Internet y las nuevas tecnologías han supuesto un modo efectivo de explotación de este ideario yihadista y propiciado que, bajo este ideario radical, diversos individuos puedan realizar actuaciones autónomas en países occidentales sin necesidad de dependencia de una estructura de mando jerarquizada ${ }^{8}$. Con lo cual, aunque la amenaza yihadista se percibe como evidente, los terroristas son cada vez más difíciles de identificar. Constituye un peligro difuso y con un alto potencial de hacer daño. Lo cual convierte a este fenómeno en algo que no puede ser combatido exclusivamente por las fuerzas de seguridad como si de un delito más se tratase, sino que representa una amenaza en la que los servicios de defensa y de inteligencia tienen un papel relevante que cumplir y en el que la cooperación con los mismos cuerpos y servicios de otros Estados se convierte en algo crucial.

Una de las mayores preocupaciones en los últimos tiempos en la lucha contra el terrorismo y la salvaguarda de la seguridad nacional ha sido precisamente frenar y perseguir a los denominados «combatientes terroristas extranjeros» ${ }^{9}$, especialmente tras la Resolución del consejo de Seguridad de naciones Unidas $2178(2014)^{10}$. Se

5 REINARES, Fernando (2016). «Amenaza yihadista en Europa, ¿qué hacer?». Estudios de Política Exterior, n. ${ }^{\circ} 171$, pág. 52.

${ }^{6} \mathrm{Al}$ respecto, véase TORRES SORIANO, Manuel (2009). La dimensión propagandística del terrorismo yihadista global, Madrid, Ministerio de Defensa.

7 A este respecto son ilustradoras las palabras del General Michael Hayden, ex director de la CIA, citadas por GONZÁLEZ CUSSAC (2016). op. cit., pág. 123: «Antes el enemigo era fácil de descubrir y difícil de destruir y ahora es más fácil acabar con él pero mucho más difícil de localizar».

8 DÍAZ MATEY, Gustavo (2016). «El papel de la inteligencia en la lucha contra el terrorismo yihadista salafista». Revista CIDOB d'Afers Internacionals, n. ${ }^{\circ} 116$, pág. 213.

9 Nacionales que viajan o intentan viajar a un Estado distinto de sus Estados de residencia o nacionalidad, y demás personas que viajan o intentan viajar desde sus territorios a un Estado distinto de sus Estados de residencia o nacionalidad, con el propósito de cometer, planificar o preparar actos terroristas o participar en ellos, o proporcionar o recibir adiestramiento con fines de terrorismo» Resolución del Consejo de Seguridad de UN 2178 (2014)

${ }_{10}$ U.N. Security Council Resolution 2178 (2014) on Foreign Terrorist Fighters 
trata de individuos que viajan y retornan a Occidente de escenarios como Siria e Irak tras haber acumulado conocimiento y experiencia en el manejo de armamento, con contactos y conocimiento de rutas y agentes facilitadores, con la intención de cometer atentados. Nos enfrentamos igualmente a la creciente amenaza que representan las personas que, aunque permanecen dentro de Europa, reciben inspiración o instrucciones de grupos terroristas situados en el extranjero.

La lucha contra los combatientes terroristas extranjeros ha conducido a la modificación de la legislación penal de algunos países. A seguir en ese camino invita la Directiva (UE) 2017/541 del Parlamento y el Consejo, de 15 de marzo de 2017, relativa a la lucha contra el terrorismo. En concreto, en España ya se incluyeron nuevos tipos delictivos que penalizan conductas que antes no lo estaban como el abandonar España con el objetivo de unirse a las filas de grupos que pretenden cometer ataques terroristas.

Pero la cuestión está no solo en penalizar, sino especialmente, en combatir el terrorismo a través de la prevención. Por ello es crucial, entre muchas otras cosas, el control de la salida y entrada de posibles terroristas yihadistas en las fronteras europeas y ahí es donde la cooperación se vuelve crucial. Naciones Unidas, con el objetivo de impedir los viajes de combatientes terroristas extranjeros desde sus territorios o a través de ellos, invitaba a un mayor intercambio de información con el propósito de identificar a dicho combatientes, comprender sus modalidades de desplazamiento y difundir las prácticas de evaluación empírica del riesgo que entrañen. Así, se decía:

«El Consejo de Seguridad reitera, según lo expresado en las resoluciones 2161 (2014) y 2178 (2014), su llamamiento a los Estados Miembros para que utilicen las bases de datos de INTERPOL y exijan que las aerolíneas de su jurisdicción proporcionen información anticipada sobre pasajeros para detectar la partida, el ingreso o el tránsito por sus territorios de personas que figuran en la lista de sanciones contra Al-Qaida y además alienta a que suministren listas de pasajeros, cuando proceda, a las autoridades nacionales competentes».

Estas afirmaciones ponían de manifiesto la consciencia sobre la utilidad del intercambio de datos para la persecución del terrorismo, al igual que de otros delitos a escala nacional y transnacional. Con similar objetivo de compartir datos, en el marco de Naciones Unidas actúa también un grupo de trabajo sobre gestión de fronteras y cumplimiento de la ley en relación con la lucha contra el terrorismo ${ }^{11}$.

En lo que respecta al ámbito de la UE, ha de recordarse igualmente que entre las prioridades legislativas para 2017, aprobadas por el Consejo el 13 de diciembre de 2016, se encontraba el «proteger mejor la seguridad de los ciudadanos de la UE, en particular mediante una mayor vigilancia de las fronteras exteriores (a través de

\footnotetext{
${ }^{11}$ https://www.un.org/counterterrorism/ctitf/es/border-management-and-law-enforcement-relatingcounter-terrorism
} 
un sistema de entradas y salidas, la implantación de «fronteras inteligentes» y un sistema europeo de información y autorización de viajes), reforzando las normas para la compra y posesión de armas de fuego, luchando contra el terrorismo ${ }^{12}$, su financiación y el blanqueo de dinero, e intercambiando información sobre nacionales de terceros países».

Lo cierto es que la gestión de las fronteras y el funcionamiento eficaz de los sistemas de tratamiento de datos en las fronteras pueden servir como mecanismo de alerta temprana. Puede fortalecer la capacidad colectiva de los Estados para detectar, prevenir y combatir el terrorismo al facilitar la cooperación entre organismos y el intercambio oportuno de información, permitiendo así adoptar de forma responsable las decisiones cruciales.

Quisiéramos abordar en este trabajo los concretos instrumentos de gestión de datos en fronteras que pueden ser útiles en esa lucha antiterrorista, porque el primer paso en inteligencia reside en la obtención de información, que luego será analizada y tratada para convertir esa información en conocimiento. Como tendremos oportunidad de comprobar, muchas de las bases de datos en fronteras se crearon para controlar la entrada de inmigrantes en las fronteras europeas, pero la información que ofrecen dichos sistemas puede servir para luchar contra este otro reto que nos amenaza, el del terrorismo yihadista. No obstante, se trata de fenómenos distintos. Es cierto que la nueva oleada de ataques yihadistas ha coincidido, en el mismo espacio temporal, con la mayor crisis migratoria a la que se ha tenido que enfrentar Europa debido a crisis humanitarias y posteriormente a la guerra de Siria u otros conflictos. Pero, no son lo mismo. El terrorismo yihadista y la inmigración poco o nada tienen que ver, por mucho que se hayan querido vincular o se hayan pretendido justificar determinadas políticas contra la inmigración como mecanismo de lucha contra el terrorismo yihadista, con el fácil argumento de que frenando la inmigración se evita la entrada de potenciales terroristas en Europa ${ }^{13}$.

\section{LA IMPORTANCIA DEL INTERCAMBIO DE DATOS SOBRE EL TRASPASO DE FRONTERAS EN EL ESPACIO SCHENGEN}

A pesar de que en el ámbito de la UE la responsabilidad principal de diseñar el sistema legal en materia de seguridad nacional y de su aplicación a nivel nacional recae en cada Estado miembro (art. 72 TFUE), la Unión se ha embarcado en la armonización de los sistemas jurídicos con el fin de lograr una mayor eficacia en la

12 El subrayado es nuestro.

13 OLESTI RALLO, Andreu (2016). «La crisis migratoria y la reinstauración de los controles de las fronteras interiores en el espacio Schengen». Revista Española de Derecho Internacional, vol. 68, 1, págs.243-247. 
lucha contra el terrorismo (art. 29 del Tratado de la UE) ${ }^{14}$, al igual que en una cooperación policial y judicial en materia penal ${ }^{15}$, regulada en el Título V del Tratado de TFUE, cuyo art. 83 se refiere explícitamente al terrorismo como forma grave de delincuencia.

La estrategia terrorista de la UE se ha sustanciado principalmente a través de la Política Exterior y de Seguridad común (PESC), el antiguo segundo pilar que sigue rigiéndose por las reglas de cooperación intergubernamental ${ }^{16}$, aunque también se ha apoyado en la política comunitaria de interior y en el Espacio Europeo de Seguridad, Libertad y Justicia (también ahí se ubicaron las políticas de control de las fronteras, asilo e inmigración), donde el papel del Parlamento y de la Comisión son más notorios $^{17}$. Como es sabido, desde la entrada en vigor del Tratado de Lisboa todo ello se «comunitarizó», desapareciendo aquel tercer pilar (arts. 66 y 67 TFUE).

En todo caso, aunque la política de la UE en materia terrorista se articula sobre la base de los principios básicos de la armonización de legislaciones, la cooperación y el reconocimiento mutuo de resoluciones judiciales, la cuestión de la seguridad sigue siendo en último extremo una cuestión fundamentalmente estatal, en cuanto que corresponde a cada estado la responsabilidad de velar por la seguridad nacional. De hecho, el art. 73 del TFUE indica que, junto a la adopción en el marco de la UE de acciones de cooperación en materia de seguridad, «los Estados

${ }^{14}$ Un desarrollo de esta cooperación puede encontrarse, entre otros, en el art. 4 de la Posición común del Consejo de 27 de diciembre de 2001, sobre the application of specific measures to combat terrorism (aplicación de medidas especiales para combatir el terrorismo) (2001/931/CFSP); la Decisión del Consejo de 28 de noviembre de 2002, que establee un mecanismo para evaluar los sistemas legales y su implementación a nivel nacional en la lucha contra el terrorismo (2002/996/JHA). Véase también la Decisión del Consejo 2003/48/ JHA, de 19 de diciembre de 2002, sobre the implementation of specific measures for police and judicial cooperation to combat terrorism in accordance with Article 4 of Common Position 2001/931/CFSP; y la Decisión Marco del Consejo de 13 de junio de 2002, sobre the European arrest warrant and the surrender procedures between Member States (2002/584/JHA).

15 En dicho ámbito se adoptaron la Decisión Marco 2008/675/JAI del Consejo, de 24 de julio de 2008, relativa a la consideración de las resoluciones condenatorias entre los Estados miembros de la UE con motivo de un nuevo proceso penal; la Decisión Marco 2008/315/JAI, de 26 de febrero de 2009, relativa a la organización y al contenido del intercambio de información de los registros de antecedentes penales entre Estados miembros; y la Decisión 2008/633/JAI sobre el acceso para consultar el Sistema de Información de Visados (VIS) por las autoridades designadas de los Estados miembros y por Europol, con fines de prevención, detección e investigación de delitos de terrorismo y otros delitos graves.

Las Decisiones citadas se implementaron en España con la aprobación de la Ley Orgánica 7/2014, sobre intercambio de antecedentes penales y consideración de resoluciones judiciales en la UE.

${ }^{16}$ Esa cooperación se ve reforzada con el art 222 del TFUE, que estipula una cláusula de solidaridad. Ésta exige la acción conjunta de todos los países de la UE en caso de que uno de ellos sea objeto de un ataque terrorista. Sin embargo, se aclara en la Declaración relativa a este art. 222 que ninguna de las disposiciones del mismo «pretende afectar al derecho de otro Estado miembro de escoger los medios más apropiados para cumplir con su obligación de solidaridad respecto de ese Estado miembro». Y a ello hay que añadir el Dispositivo Integrado de Respuesta Política de la UE a las Crisis (DIRPC) adoptado en 2013.

${ }_{17}$ En realidad, esas medidas se repartieron entre el título IV del Tratado de la Comunidad Europea y el antiguo tercer pilar, la Cooperación policial y judicial en materia penal. 
miembros tendrán la posibilidad de organizar entre ellos y bajo su responsabilidad formas de cooperación y coordinación en la medida en que lo estimen apropiado, entre los servicios competentes de sus administraciones responsables de velar por la seguridad nacional».

Aun así, en la lucha antiterrorista, la necesidad de cooperación entre servicios de seguridad y agencias de inteligencia se ha ido convirtiendo en una cuestión recurrente en la $\mathrm{UE}^{18}$. Porque la prevención de nuevos ataques terroristas puede reforzarse, entre otras cosas, mediante el establecimiento de programas de intercambio de información sobre delincuentes, fronteras, transacciones financieras, desplazamientos, etc. Ya que, todas las medidas proactivas, entre las que se incluye la labor de los servicios de inteligencia y las fuerzas de seguridad para anticipar y contener futuros atentados, deben partir del conocimiento de los patrones que están desarrollando los «nuevos» terroristas $^{19}$. Hablamos de la cooperación entre las agencias de seguridad y defensa, generalmente más ocupadas de la seguridad exterior, y de los cuerpos de seguridad, tradicionalmente más centrados en la seguridad interior, porque la lucha contra el terrorismo es un buen ejemplo de lo difícil que es mantener esa habitual separación entre seguridad interior y seguridad exterior.

Con este propósito de favorecer el intercambio de información del que hablábamos, en el área Schengen ${ }^{20}$ se aprobó la Decisión Marco del Consejo 2006/960/JAI, que estableció un sistema simplificado de intercambio de información e inteligencia entre los servicios de seguridad de los Estados miembros de la Unión Europea (UE) a través de Europol $^{21}$ y Eurojust. Es el que se conoce como Sistema de Información de Schengen (SIS). Haciendo uso de dicha información se puede hacer llegar a los servicios

${ }^{18} \mathrm{La}$ necesidad de coordinar de forma muy intensa la protección de los datos debido a la globalización se ha hecho absolutamente evidente en otros muchos ámbitos. La llamada a un ejercicio cooperativo en materia de datos es algo crucial hoy, como indica Artemi Rallo. Véase, RALLO LOMBARTE, Artemi (2015). «Cooperación y coordinación entre autoridades de protección de datos», en Artemio Rallo y Rosario García (Ed.), Hacia un nuevo Derecho europeo de protección de datos, Valencia, Tirant lo Blanch, pág. 740.

19 DÍAZ MATEY, Gustavo (2016). «El papel de la inteligencia en la lucha contra el terrorismo yihadista salafista». Revista CIDOB d'Afers Internacionals, n. ${ }^{\circ} 116$, pág. 209.

${ }^{20}$ El Acuerdo de Schengen — por el que se establece la supresión de las fronteras comunes entre los países que integran su espacio y se crean controles comunes en las fronteras exteriores de estos- no incluye a todos los países de la UE. Hay países que no son de la UE pero están en el espacio de Schengen (Islandia, Liechtenstein, Noruega y Suiza) y hay otros que estando dentro de la UE, no lo están en el espacio de Schengen (territorios franceses de ultramar, Irlanda y Reino Unido)

${ }^{21}$ El art. 88 del TFUE (antiguo art. 30 TUE) establece que «(l)a función de Europol es apoyar y reforzar la actuación de las autoridades policiales y de los demás servicios con funciones coercitivas de los Estados miembros, así como su colaboración mutua en la prevención de la delincuencia grave que afecte a dos o más Estados miembros, del terrorismo y de las formas de delincuencia que lesionen un interés común que sea objeto de una política de la Unión, así como en la lucha en contra de ellos». Y se indica que entre las actividades que mediante reglamento se le pueden asignar está la recogida, almacenamiento, tratamiento, análisis e intercambio de la información, en particular la transmitida por las autoridades de los Estados miembros o de terceros países o terceras instancias. 
de seguridad de los países miembros alertas y avisos, entre otras cosas, sobre la entrada de sospechosos terroristas u otros delincuentes, o información sobre riesgos de comisión de atentados, etc. Esta información ha servido para evitar un número muy considerable de ataques terroristas, aunque no siempre el intercambio de información constituye una garantía de ello. Lo que sí es cierto es que cuando se han producido atentados, la sombra de una falta de cooperación o de intercambio de información ha estado siempre presente en el debate público ${ }^{22}$.

Otro instrumento que ha servido para facilitar la cooperación en materia de datos de tránsito entre las autoridades de los Estados miembros y países asociados al espacio Schengen ha sido Frontex. Frontex es la Agencia Europea para la gestión de la cooperación operativa en las fronteras exteriores de la UE que nació en $2004^{23}$ para facilitar a los Estados miembros y los países asociados al espacio Schengen a controla las fronteras exteriores y a armonizar los controles fronterizos, facilitando la cooperación entre las autoridades fronterizas de cada país de la UE y suponiendo una herramienta de intercambio de conocimiento técnico y experiencia. Aunque Frontex nació efectivamente como una operación para controlar los flujos migratorios, se ha extendido a otras amenazas de seguridad como puede ser la trata de seres humanos o como es el terrorismo internacional. Entre sus diferentes actividades, (insistimos) especialmente orientadas al control de flujos migratorios, la Agencia lleva a cabo actividades de análisis de riesgos y sus conclusiones se trasladan a los países de la UE y la Comisión. Igualmente desarrolla y utiliza sistemas informativos que permiten un intercambio rápido de información entre las autoridades fronterizas. Frontex lo que hace es actuar como coordinadora, tendiendo puentes entre las autoridades fronterizas.

Esa información sin duda puede ser relevante para la lucha contra el terrorismo yihadista. De hecho, este tema fue uno de los que ocupó la reunión de Frontex África que tuvo lugar en Las Palmas de Gran Canaria el 23 de mayo de 2017, evento que reunió a la Agencia con 28 países africanos, directores de inteligencia y expertos en el área de fronteras. La idea de organizar estas reuniones entre Frotex y los países africanos surgió en 2010 por la intensidad de los flujos migratorios desde este continente, pero también por el avance del terrorismo en Nigeria, Niger, Mali y Burkina Faso, el auge del terrorismo yihadista salafista en el Magreb, los ataques perpetrados en Túnez o el origen norteafricano de los autores de algunos ataques terroristas, que se han convertido en otros de los temas abordados en esos encuentros.

${ }^{22}$ Entre otros ejemplos, se indicó que, en los atentados de Londres de junio de 2017, los sospechosos habían estado en las pantallas de los Servicios de inteligencia o habían sido investigados por conexiones terroristas. Un flujo anticipado de información entre distintos servicios hubiese facilitado las labores de prevención. DÍAZ MATEY. op. cit., pág. 221. Pero esos mismos fallos de coordinación entre servicios de inteligencia saltaron a la luz tras el 11-S

23 Se crea Frontex en virtud del Reglamento (CE) n. . 2007/2004 del Consejo, por el que se crea una Agencia Europea para la gestión de la cooperación operativa en las fronteras exteriores de los Estados miembros de la UE. 
Con la misma intención de convergencia y canje de información se acordó el Tratado Prüm en 2005 entre varios países de la UE ${ }^{24}$. En este se estableció un marco legal para profundizar la cooperación entre los Estados miembros en la lucha contra el terrorismo ${ }^{25}$, la delincuencia transfronteriza y la inmigración ilegal, contenidos que fueron incorporados posteriormente a la Decisión del Consejo de 2008 sobre el mismo objetivo y extendidos a todos los Estados del área ${ }^{26}$. Aquel supuso una profundización en los pasos dados en el marco Schengen, de ahí que a este Tratado se le conozca también como Schengen III $^{27}$. Más concretamente, en él se prevé el intercambio entre las Partes contratantes de perfiles de ADN, datos dactiloscópicos, registros de matriculación de vehículos y otros datos de carácter personal. El tratado permite a las autoridades de los demás Estados parte un acceso inmediato a la información que conste en los mismos, mediante índices de referencia, a través de puntos de contacto. Aunque el Tratado tiene naturaleza internacional, en todo caso, establece su compatibilidad con las normas europeas al prever, en su artículo 47, que las disposiciones del Tratado solo serán aplicables en la medida en que sean compatibles con el derecho de la UE. Se establece, además, que si en el futuro la Unión adoptara normas que puedan afectar al ámbito de aplicación del Tratado, las disposiciones del mismo dejarán de aplicarse en beneficio del derecho de la UE. La utilidad de este marco ha quedado demostrada. De hecho, las Decisiones Prüm fueron una herramienta que sirvió a los investigadores franceses tras los atentados terroristas de París de noviembre de 2015.

Por lo que decíamos al comienzo de este trabajo sobre la excesiva relación que parece evidenciarse entre terrorismo e inmigración, hay que recordar que precisamente una de las críticas que se hizo en su día al Tratado de Prüm es la vinculación que se produce entre la inmigración ilegal, el terrorismo y la criminalidad organizada ${ }^{28}$, algo que es preocupante y que, sin embargo, continúa siendo una idea recurrente.

El caso es que, paso a paso, el control de la entrada y salida de potenciales terroristas o delincuentes ha ido materializándose en nuevas herramientas como,

24 El Tratado de Prüm, de profundización en la cooperación transfronteriza, en particular en materia de la lucha contra el terrorismo, la delincuencia transfronteriza y la migración ilegal, se concluyó el 27 de mayo e 2005, entrando en vigor el 1 de noviembre de 2016. Fue firmado por Bélgica, Alemania, España, Francia, Luxemburgo, Países Bajos y Austria. Con el tiempo se han ido incorporando más países.

25 REMOTTI CARBONELL, Carlos (2007). «Las medidas contra el terrorismo en el marco del Tratado de Prüm». Revista de Derecho Constitucional Europeo, n. ${ }^{\circ}$, págs. 184-203.

26 Decisión 2008/616/JAI del Consejo, de 23 de junio de 2008, relativa a la ejecución de la Decisión 2008/615/JAI sobre la profundización de la cooperación transfronteriza, en particular en materia de lucha contra el terrorismo y la delincuencia transfronteriza.

27 Como indicaba Gregorio Cámara, la pretensión de Prüm fue avanzar de manera mucho más rápida en el intercambio de datos e información para cubrir los objetivos descritos en la lucha contraterrorista, la delincuencia transfronteriza y la inmigración ilegal. Pero lo hizo orillando el marco comunitario y generando un nuevo nivel de cooperación en estas materias entre los Estados firmantes. CÁMARA VILLAR, Gregorio (2007). «La garantía de los derechos fundamentales afectados por la Convención de Prüm». ReDCE, n. ${ }^{\circ} 7$, págs. 99.

28 CÁMARA VILLAR, Gregorio, Ibidem, pág. 116. 
por ejemplo, la que entró en funcionamiento en 2014, el Sistema de Información de Visados (VIS), aplicable a los solicitantes de visado Schengen de corta duración. El VIS está conectado a todos los consulados de los Estados Schengen encargados de la expedición de visados y a todos los puntos de cruce de las fronteras exteriores. En estos pasos fronterizos, el VIS permite a la guardia de fronteras comprobar que el titular de un visado biométrico es efectivamente la misma persona que lo solicitó. Las autoridades policiales y Europol tienen acceso al sistema para fines de detección e investigación de delitos de terrorismo y otros delitos graves. En octubre de 2011 el VIS comenzó a funcionar en todos los consulados de Estados Schengen encargados de la expedición de visados en el norte de África, y ahora se ha extendido al resto del mundo.

De todos modos, también hay que reconocer que, a pesar de estas previsiones y los pasos hacia la cooperación, como decíamos, los centros de inteligencia estatales tradicionalmente han tendido a trabajar en clave doméstica y han recelado de compartir toda la información. Pero, por la fuerza de los hechos, ha sido necesario ir rompiendo la renuencia de muchos Estados a compartir sus datos en ese terreno. Esta tendencia ha ido cambiando con el tiempo y, desde luego, recibió un fuerte espaldarazo en buena medida debido a la reiteración de ataques terroristas que Europa empezó a sufrir a partir de $2015^{29}$, cuando parecía que los atentados de Madrid (2004) y Londres (2005) empezaban a ser un mal sueño de una década atrás ${ }^{30}$. Las advertencias que los servicios policiales y los analistas venían realizando sobre el peligro de la extensión al continente europeo de actos terroristas promovidos por grupos yihadistas se hicieron realidad de forma dramática ${ }^{31}$. Tanto es así que, tras los atentados de marzo de 2016 el concepto de «Security Union» fue lanzado como un modo de virar de la cooperación para la protección de la seguridad nacional interna de cada uno de los Estados hacia la idea de proteger la seguridad colectiva de la Unión como conjunto ${ }^{32}$.

29 BARNES, Julian E. y FIDLER, Stephen (2016). «Brussels Attacks give new impetus for more intelligence Sharing in Europe». The Wall Street Journal (18 de abril de 2016) (en línea) https://www. wsj.com/articles/brussels-attacks-give-new-impetus-for-more-intelligence-sharing-ineurope-1460952001

${ }^{30}$ La respuesta por oleadas al fenómeno terrorista es algo característico de la UE, consistente en la reacción reforzada solo tras cada ocasión en que el fenómeno terrorista se vuelve más activo. COOLSAET, R. (2010). «EU counterterrorism strategy: value added or chimera?». International Affairs (Chatam House, The Royal Institute of International Affairs), Vol. 86, Issue 4, julio 2010, págs.857-873; en concreto, pág. 869.

31 AA.VV. (2016). "La amenaza del terrorismo yihadista». Cuadernos del centro memorial de las víctimas del terrorismo, n. ${ }^{\circ} 1$, págs. 27-46.

32 European Commission. «Communication from the Commission to the European Parliament, the European Council and the Council - delivering on the European Agenda on Security to fight against terrorism and pave the way towards an effective and genuine Security Union», COM (2016) 230 final, 20 April 2016, pp. 2 y 9. 
La necesidad condujo a una reacción en el marco político y normativo de la UE, y también en el doméstico, con la introducción de medidas y herramientas que iremos analizando en los siguientes apartados.

En lo que respecta al espacio Schengen, que es de lo que veníamos hablando en este apartado, en 2016 se aprobó el Reglamento por el que se establece lo que se conoce como Código de Fronteras Schengen ${ }^{33}$, que constituye un código de normas de la Unión para el cruce de personas por las fronteras. Estamos de nuevo ante un ejemplo de norma que, aunque ella misma indica que puede servir para la prevención de cualquier amenaza a la seguridad interior de los Estados ${ }^{34}$, sin duda, tiene como objetivo principal contribuir al control de la inmigración.

Ese Código Europeo de Fronteras ha sido recientemente reformado por el Reglamento que modifica el Código Europeo de Fronteras, aprobado en marzo de $2017^{35}$, que ha procedido a actualizar dicho Código justificándolo en las nuevas necesidades de la UE y sus Estados miembros, en relación con demandas de mayor seguridad y adecuado control de la inmigración. De nuevo los conceptos seguridad e inmigración vuelven a aparecer vinculados. De forma resumida, cabe destacar que la reforma obliga a los Estados miembros a:

a) Llevar a cabo controles sistemáticos, mediante la comprobación en las bases de datos pertinentes, de todas las personas, incluidas aquellas que disfrutan del derecho a la libre circulación conforme al Derecho de la Unión (es decir, los ciudadanos de la UE y los miembros de sus familias que no sean ciudadanos de la UE) cuando crucen las fronteras exteriores. Esta opción no cabía hasta ahora, puesto que previamente los Estados miembros estaban obligados a someter a controles sistemáticos solo a los nacionales de terceros países que no tuvieran derecho a la libre circulación de conformidad con las disposiciones pertinentes de la UE, realizando además las comprobaciones oportunas en las bases de datos correspondientes.

b) Además, desde la regulación que comentamos, los datos de las personas que crucen la frontera exterior se contrastarán con los que figuran en bases de datos de interés policial, (por ejemplo, el Sistema de Información Schengen

33 Reglamento (UE) 2016/399 del Parlamento Europeo y del Consejo, de 9 de marzo de 2016 por el que se establece un Código de normas de la Unión para el cruce de personas por las fronteras (Código de fronteras Schengen)

${ }^{34}$ Se indica en el considerando 6 que «(e)l control fronterizo debe contribuir a la lucha contra la inmigración clandestina y la trata de seres humanos, así como a la prevención de cualquier amenaza a la seguridad interior, al orden público, a la salud pública y a las relaciones internacionales de los Estados miembros». Y el considerando 7 que dichos controles incluyen también el análisis de los riesgos para la seguridad interior y de las amenazas que pueden afectar la seguridad de las fronteras exteriores. Idem en considerando 22 .

35 Reglamento (UE) 2017/458, del Parlamento Europeo y del Consejo de 15 de marzo de 2017, por el que se modifica el Reglamento (UE) 2016/399, en lo relativo al refuerzo de los controles mediante la comprobación en las bases de datos pertinentes en las fronteras exteriores. Entró en vigor el 7 de abril de 2017. 
o la base de datos de Interpol sobre documentos de viaje extraviados o sustraídos). La obligación se aplicará a la entrada y salida de todas las fronteras exteriores del Espacio Schengen, es decir, terrestres, aéreas y marítimas.

Una vez más, insistimos en que muchas de las medidas adoptadas para asegurar las fronteras exteriores, pese a que se alega que constituyen instrumentos de salvaguarda de la seguridad nacional, no tienen a esta como principal objetivo. En gran parte, su fin principal es controlar las fronteras para frenar la inmigración, pero la potencial efectividad para la lucha antiterrorista queda menos demostrada ${ }^{36}$.

En esa misma línea de fomentar el intercambio de datos sobre cruce de fronteras, recientemente la Comisión Europea propuso la creación de un Sistema de Entradas y Salidas $(\mathrm{SES})^{37}$, que será un sistema de captación de datos sobre la entrada de nacionales de terceros países en el espacio Schengen, tengan o no la obligación de visado. El pasado 20 de noviembre de 2017 el Consejo adoptó finalmente el Reglamento para el establecimiento de ese Sistema de Entradas y Salidas ${ }^{38}$ y el Reglamento por el que se modifica el Código de fronteras Schengen en relación con el Sistema de Entradas y Salidas ${ }^{39}$.

El primero permitirá el establecimiento de un registro de historiales de viaje de nacionales de terceros países, incluidos los sospechosos de haber cometido delitos y los autores o víctimas de delitos. Un registro que será accesible para las autoridades de los Estados europeos, especialmente cuando el acceso se hace con fines policiales y de prevención, detección e investigación de delitos de terrorismo u otros graves (considerando 22). Por lo tanto, completará la información del SIS. El Consejo y el Parlamento Europeo deben ahora firmar el Reglamento adoptado. El texto firmado se publicará en el Diario Oficial y entrará en vigor veinte días después.

Pese a que el SES pueda presentarse como una herramienta que puede contribuir a la lucha contra el terrorismo, tiene una evidente intención principal de controlar o gestionar las fronteras exteriores del espacio Schengen para fiscalizar a su vez los flujos migratorios. Esa misma finalidad es la que persigue el nuevo Reglamento por el que se modifica el Código de Fronteras Schengen. De hecho, respecto a los programas nacionales de facilitación de entrada ${ }^{40}$, que ya contemplaba el Código de Fronteras

36 WENSINK, PwC y otros (2017). The European Union's Policies on Counter-Terrorism Relevance, Coherence and Effectiveness, Study for the libe Committee. Bruselas, European Parliament, 2017, pág. 57.

37 Comisión Europea. «Fronteras más sólidas e inteligentes en la UE», Bruselas, 6 de abril de 2016.

38 Reglamento del Parlamento Europeo y del Consejo por el que se establece un Sistema de Entradas y Salidas (SES) para registrar los datos de entrada y salida y de denegación de entrada relativos a nacionales de terceros países que crucen las fronteras exteriores de los Estados miembros, se determinan las condiciones de acceso al SES con fines policiales y se modifican el Convenio de aplicación del Acuerdo de Schengen y los Reglamentos (CE) n. 767/2008 y (UE) n. 1077/2011.

39 Reglamento del Parlamento Europeo y del Consejo por el que se modifica el Reglamento (UE) 2016/399 en lo que respecta a la utilización del Sistema de Entradas y Salidas

${ }^{40}$ Cada Estado miembro podrá establecer un programa voluntario («programa nacional de facilitación») con el fin de permitir que los nacionales de un tercer país, o los nacionales de un tercer país específico, que no gocen del derecho de libre circulación en virtud del Derecho de la Unión se beneficien de las facilidades a que se refiere el apartado 2 al cruzar la frontera exterior de un Estado miembro. 
Schengen, se indica que la guardia de fronteras deberá evaluar si se impide la entrada en dicho programa de facilitación a aquellos individuos que presenten «algún riesgo para la seguridad de cualquier Estado miembro» (art. 8 quinquies). También se establece que, en determinados casos, los nacionales de terceros países deben facilitar datos biométricos a efectos de las inspecciones fronterizas. Si un nacional de un tercer país se niega a facilitar datos biométricos para la creación de su expediente individual o para la realización de inspecciones fronterizas, ha de adoptarse una decisión de denegación de entrada.

Ahora mismo se encuentra también en estudio el RTP o Programa de Viajeros Registrados, que permitiría una rápida entrada en la UE a los viajeros frecuentes pre-aprobados de terceros Estados y que será puesto en relación con el Sistema de Información de Visados (VIS). Algunos países individualmente ya tienen su propio RTP firmado con terceros países ajenos a la UE (Países Bajos, Alemania, Finlandia.... ${ }^{41}$.

Por último, cabe citar que el 9 de junio de 2017, el Consejo acordó asimismo una orientación general sobre la propuesta de Sistema Europeo de Información y Autorización de Viajes (SAIV). La orientación general constituye la posición del Consejo para las negociaciones con el Parlamento Europeo, una medida que tendrá claramente una finalidad más orientada a frenar la inmigración ilegal que a la seguridad, pues lo que el SAIV permitirá es realizar controles anticipados y, en caso necesario, denegar la autorización de viaje a los nacionales de terceros países exentos de visado que viajen al espacio Schengen ${ }^{42}$. Otro mecanismo que puede ayudar al mantenimiento de la seguridad nacional, pero que, evidentemente tiene un fin más centrado en el control de la inmigración.

\section{LAS NUEVAS DIRECTIVAS SOBRE EL TRATAMIENTO DE DATOS Y LA SALVAGUARDA DE LA SEGURIDAD NACIONAL}

Como decíamos anteriormente, los atentados de París de 2015 y de Bruselas en 2016 empujaron a los Estados de la UE y a sus instituciones a reevaluar su política de seguridad, y proponer nuevas iniciativas para reforzar la lucha antiterrorista, en concreto en el establecimiento de controles sistemáticos de fronteras, control de armas y registro de pasajeros. Precisamente esos atentados aceleraron la adopción de la Directiva 2016/680, de 27 de abril, sobre el tratamiento de datos por las autoridades en el ámbito de la prevención y persecución del crimen ${ }^{43}$, al igual que el nuevo Regla-

41 RUÍZ DOMÍNGUEZ, Fernando (2016). «Control de fronteras y tráfico de pasajeros aéreos: ¿seguridad vs libertad?». Documento Opinión 71/2016, Ieee.es, de 12 de julio, págs. 1-18.

42 El sistema se aplicará a los nacionales de terceros países exentos de visado, así como a aquellos que están exentos de la obligación de visado para el tránsito aeroportuario. Antes de iniciar su viaje tendrán que obtener una autorización para viajar mediante una solicitud en línea.

43 Directive (EU) 2016/680 of the European Parliament and of the Council of 27 April 2016 on the protection of natural persons with regard to the processing of personal data by competent authorities 
mento 2016/679 de Protección de Datos ${ }^{44}$. Asimismo, la Comisión Europea presentó en diciembre de ese mismo año un Proyecto de Directiva de lucha antiterrorista ${ }^{45}$, que vendría a sustituir a la Decisión marco 2001/475/JHA sobre la misma materia, y que fue aprobada el 15 de marzo de $2017^{46}$. Aunque esta última Directiva se ocupa fundamentalmente de la respuesta de la justicia penal de los Estados miembros a los delitos de terrorismo, también sirve como punto de referencia para el intercambio de información entre las autoridades nacionales y resalta la importancia de las pruebas electrónicas, al hacer hincapié en el carácter transfronterizo del terrorismo yihadista y la necesidad de una respuesta global y coordinada al mismo.

La nueva Directiva 2016/680 sobre tratamiento de datos en la prevención y persecución del crimen, mencionada arriba, y que debía implementarse en dos años desde su publicación, abre un nuevo escenario más reforzado en el ámbito del intercambio de datos entre Estados. La Directiva se aplicará tanto al procesamiento transfronterizo de datos personales, como al tratamiento de datos personales por parte de las autoridades policiales y judiciales a nivel puramente estatal. Ha de tenerse en cuenta que la anterior Decisión Marco de 2008, que queda sustituida por la Directiva, sólo cubría el intercambio transfronterizo de datos. La nueva Directiva vuelve a hacer hincapié en la creciente necesidad de intercambiar datos para la prevención y la lucha contra la delincuencia transnacional y el terrorismo. Pero, igualmente, subraya la obligación de garantizar el cumplimiento de los principios básicos que regulan la protección de datos personales (Considerando n. ${ }^{\circ}$ ). De hecho, establece normas mínimas que han de respetarse en el tratamiento de datos dentro de cada Estado miembro, se establecen derechos concretos y se fijan limites en el flujo de datos.

\section{EL REGISTRO DE PASAJEROS (EL PERSONAL NAME RECORD O PNR)}

Entre las medidas de intercambio de información de personas que cruzan fronteras, una cuestión muy debatida fue una propuesta, que llevaba estancada desde 2013 , de establecer un Registro de pasajeros aéreos que podría compartirse entre los Estados

for the purposes of the prevention, investigation, detection or prosecution of criminal offences or the execution of criminal penalties, and on the free movement of such data, and repealing Council Framework Decision 2008/977/JHA. Entró en vigor el 5 de mayo de 2016, aunque los Estados miembros disponían hasta el 6 de mayo de 2018 para implementarla.

${ }^{44}$ Regulation (EU) 2016/679 of the European Parliament and of the Council of 27 April 2016 on the protection of natural persons with regard to the processing of personal data and on the free movement of such data, and repealing Directive 95/46/EC (General Data Protection Regulation). Entró en vigor el 24 de mayo de 2016, pero empezó a aplicarse a partir del 25 de mayo de 2018.

${ }^{45}$ COM (2015) 625 final, 2015/0281 (COD) Brussels, 2.12.2015.

46 Directiva (UE) 2017/541, del Parlamento Europeo y del Consejo, relativa a la lucha contra el terrorismo. 
de la UE —el PNR (Personal Name Record) —. El Parlamento Europeo mostró reiteradamente su preocupación por el daño que podría generar para la privacidad de los viajeros $^{47}$.

A pesar de ello, y de nuevo tras los atentados de Paris de noviembre de 2015, en el Consejo de Ministros de Interior de 4 de diciembre de ese año, se dio luz verde a la aprobación del citado Registro, que quedó finalmente regulado por la Directiva reguladora del uso del PNR para la prevención, investigación y persecución del terrorismo y otros crímenes graves, aprobada el 21 de abril de $2016^{48}$. La Directiva se aplicará a los vuelos que tienen origen o destino fuera de la UE, pero los Estados miembros también podría extenderlo a los domésticos («dentro de la UE»). Ello obliga a la transmisión por parte de los prestadores de servicios de vuelo a las autoridades de una serie de datos de los pasajeros de vuelos que se obtienen en el momento de efectuar la reserva de vuelo (nombre, dirección, itinerario, si se viaja solo o acompañado, modo de pago, tarjetas bancarias utilizadas, números de teléfono, información sobre equipaje, fechas de viajes, frecuencia con que vuela... ${ }^{49}$ ).

47 Así, en abril de 2013, el Parlamento Europeo rechazó una propuesta para crear y utilizar, a nivel de la UE, un Registro PNR por sus dudas sobre su necesidad y sus preocupaciones sobre la privacidad y los datos de los ciudadanos europeos.

48 Directive(EU) 2016/681, regulating the use of Passenger Name Record (PNR) data in the EU for the prevention, detection, investigation and prosecution of terrorist offences and serious crime.

49 Los datos que quedarán registrados son los siguientes:

1. Nombre y apellidos

Es el dato esencial del registro, aunque este se «enmascarará» automáticamente a los seis meses.

2. Fecha del viaje

Esencial para seguir la pista de posibles terroristas.

3. Fecha de la reserva o emisión del billete

4. Localizador

La reserva se identifica mediante este número alfanumérico.

5. Datos del viajero

Número de teléfono, correo electrónico.

6. Destino y origen

Ahora no se puede seguir el rastro de un viajero que vuelva a Europa desde Siria a través de uno de los países habituales de escala.

7. Otros viajes

Datos sobre vuelos o desplazamientos anteriores del viajero.

8. Dirección de contacto

El dato no se enmascara a los seis meses como la identidad.

9. Medio de pago

Si el billete se pagó en efectivo o con tarjeta. En este caso se puede seguir el rastro de la tarjeta.

10. Asiento en el vuelo

11. Si viaja solo o en grupo

Un viaje organizado y programado para un grupo grande de personas permite descartar a personajes sospechosos de estar planeando un atentado.

12. Datos sobre el billete

Tarifa, viaje de ida o vuelta, fecha de compra.

13. Intermediarios 
El PNR es distinto del API (Advancce Passenger Information) que son unos datos que algunos países del mundo solicitan y sirven para identificar terroristas y criminales «conocidos» mediante el uso de alertas, mientras el registro PNR permite la evaluación del riesgo de las personas desconocidas. Se establecerán determinados «indicadores de riesgo», por ejemplo, si la reserva se ha realizado a través de Internet o por una agencia de viajes, si el billete se ha pagado en efectivo o con tarjeta de crédito, y si la ruta del pasajero sigue algún patrón habitualmente utilizado por terroristas. El fin es hacer un seguimiento de ciertos pasajeros sospechosos y poder en su caso prevenir una amenaza y anticiparse a la comisión de ciertos delitos.

Con objeto de proteger los derechos fundamentales relativos a la protección de los datos personales, a la intimidad y a la no discriminación, la Directiva incluye una serie de limitaciones de la transferencia, el tratamiento y la conservación de los datos PNR: En primer lugar, la Directiva prohibe la recogida y el uso de datos sensibles. Además, los datos PNR solo pueden conservarse durante un periodo de cinco años y deben despersonalizarse pasados seis meses de forma que el interesado deje de ser identificable inmediatamente. Igualmente, los pasajeros tienen derecho a ser informados de forma clara sobre la recogida de esos datos y de los derechos que les asisten. La Directiva exige igualmente el establecimiento de mecanismos de seguridad de datos, cuya protección quedarán en manos de una unidad de información sobre los pasajeros y un responsable de la protección de datos. Se incluyen asimismo previsiones sobre la cesión de esos datos, indicándose que la transferencia de datos PNR a terceros países solo podrá producirse en circunstancias muy particulares y deberá estudiarse caso por caso, y exclusivamente para los fines específicos de "prevenir, detectar, investigar y procesar los delitos de terrorismo o delitos graves». Por último, se indica que el tratamiento automatizado de los datos PNR no podrá ser la única base para tomar decisiones que tengan consecuencias jurídicas adversas o que afecten gravemente a una persona.

La cuestión está en cómo se va a producir la implementación de esta Directiva con el máximo respeto a la privacidad de los individuos. Se ha señalado que el proceso de trasposición va a ritmo lento en los países europeos y, obviamente, el PNR solo tendrá sentido si lo aplican todos o el mayor número de estados del espacio Schengen. Por lo que respecta a España, se cuenta con el art. 25 de la Ley de Seguridad Ciudadana, que habla de la creación de un fichero de viajeros y que fue introducido por una enmienda presentada por el Partido Popular. La enmienda supuso la inclusión del

Agencia de viajes o medio de adquisición del billete

14. Especificar si el viajero hizo uso o no de su billete

15. Equipajes

16. Reservas

Conocer si se realizó alguna modificación en la reserva del vuelo.

17. Código compartido

18. Datos generales

19. Cambios en las 18 medidas citadas 
citado art. 25, que establece que «las personas físicas o jurídicas que ejerzan actividades relevantes para la seguridad ciudadana, como las de hospedaje, trasporte de personas...quedarán sujetas a las obligaciones de registro documental e información en los términos que establezcan las disposiciones aplicables» Y lo mismo se prevé para los titulares de embarcaciones de alta velocidad, así como los de aeronaves ligeras. Como se puso de manifiesto, esta medida era necesaria para la creación, bajo la dependencia del Ministerio del Interior, del Sistema Nacional de Registro de datos de pasajeros que en aquel momento se estaba impulsando en el marco de la UE, una medida pensada para la prevención, detección, investigación y enjuiciamiento de delitos de terrorismo y otros delitos graves. No obstante, con esa previsión legal no basta y para cumplir efectivamente con la Directiva finalmente aprobada en 2016, el gobierno español tendría que adaptar debidamente la legislación española al PNR del que habla aquélla, como medida, al menos en el papel, para blindar las fronteras comunitarias al acceso de yihadistas procedentes de lugares en conflicto. Pensemos que algunos de los atentados terroristas, como el de Manchester, se perpetró por individuos que habían realizado una estancia en esas zonas de crisis (Libia, en ese caso) donde fueron entrenados.

Obviamente el registro PNR exige también la puesta en marcha de las herramientas tecnológicas necesarias que permitan el tratamiento de todos esos datos.

\section{CUANDO LOS DATOS CRUZAN LAS FRONTERAS EXTERNAS. EL INTERCAMBIO DE DATOS CON TERCEROS PAÍSES AJENOS A LA UE}

\subsection{El fallido Acuerdo PNR con Canadá y las exigencias del tribunal de justicia en materia de cesión de datos de pasajeros}

La evolución de la sociedad de la información y del conocimiento y la progresiva globalización han impuesto la necesidad de fortalecer los instrumentos de cooperación entre las autoridades nacionales en materia de datos superando el marco originario europeo $^{50}$. Un acuerdo de transmisión de datos de pasajeros de vuelos se firmó en 2006 con EEUU, que suponía transferir información sobre 34 items de los pasajeros que viniendo de Europa hicieran escala o tuvieran destino en un aeropuerto estadounidense, un acuerdo que permitía su almacenamiento durante más de 5 años. Las condiciones del acuerdo suscitaron muchas críticas, llevando a una revisión de las mismas en 2012 que condujo a una reducción en el número de ítems que se comparten a 19 epígrafes ${ }^{51}$.

50 RALLO LOMBARTE, Artemi (2015). Op. Cit., pág. 747.

51 Decisión del Consejo de 26 de abril de 2012 relativa a la celebración del Acuerdo entre los EEUU y la UE sobre la utilización y la trasferencia de los registros de nombres de los pasajeros al Departamento de Seguridad del Territorio Nacional de los EEUU. 
También se mantiene un acuerdo de intercambio de datos de pasajeros con Australia desde 2012.

Con idéntico interés, Canadá y la UE negociaron desde 2010 un acuerdo de esta misma naturaleza sobre el tratamiento y la transferencia de los datos del registro de nombres de los pasajeros (Acuerdo sobre el PNR). Tras la firma del acuerdo en 2014, el Parlamento Europeo solicitó un dictamen al TUE para saber si era conforme al Derecho de la Unión. La respuesta del TJUE llegó en su sentencia de 26 de julio de 2017 , donde consideró que ese sistema de intercambio de datos supone una «injerencia en el derecho fundamental al respeto a la vida privada» y a «la protección de los datos de carácter personal». El tribunal reconoció que esas injerencias en los derechos pueden estar justificadas «en aras de un objetivo de interés general», pero censuró que las disposiciones del acuerdo «no se limitan a lo estrictamente necesario y no establecen normas claras y precisas». En particular, no existían garantías suficientes en lo relativo a los datos «sensibles», es decir, aquellos que revelan información sobre «el origen racial o étnico, las opiniones políticas, las convicciones religiosas o filosóficas, la pertenencia a sindicatos o los relativos «a la salud o a la sexualidad».

Los magistrados entendieron, además, que en lo relativo a la conservación de los datos por parte de las autoridades canadienses, la medida de conservación no se justificaba una vez que hubieran abandonado el país «los pasajeros aéreos respecto de los cuales no se hubiese identificado ningún riesgo en materia de terrorismo o de delincuencia grave de carácter trasnacional a su llegada a Canadá».

La sentencia consideró que el acuerdo también debía revisar la «claridad y precisión» de los datos a transferir, disponer que los «modelos y criterios» utilizados son «específicos, fiables y no discriminatorios» y garantizar que los datos utilizados por Canadá se circunscriban a la prevención antiterrorista y de delitos internacionales graves. Asimismo, la Corte requirió que Canadá solo pueda comunicar esos datos a un tercer país no miembro de la UE si este tiene un acuerdo previo con la Unión y debe establecer un «derecho del pasajero» en caso de que se las autoridades no respeten la normativa. Por todo ello, los jueces del TJUE consideraron que «el acuerdo previsto no puede celebrarse en su forma actual».

\subsection{El intercambio de datos con Estados Unidos. El EU-US Umbrella Agreement y el Privacy Shield ${ }^{2}$}

Asimismo, en relación con el intercambio de datos con terceros países, procede recordar que el Tribunal de Justicia de la UE volvió a jugar un papel importante en la salvaguarda de datos personales, pues su decisión en el Asunto Digital Ireland

52 A este respecto véase un estudio más amplio en LÓPEZ AGUILAR, Fernando (2017). «La protección de datos personales en la más reciente jurisprudencia del TJUE: Los derechos de la CDFUE como parámetro de validez del Derecho Europeo, y su impacto en la relación transatlántica UE-EEUU». Teoría y Realidad Constitucional, n. ${ }^{\circ}$ 39, págs. 557-581. 
$(2014)^{53}$ conllevó la negociación y conclusión del denominado «Umbrella Agreement» de $2016^{54}$, como refuerzo de la protección de datos personales de los europeos en su transmisión de los mismos a los EEUU a efectos de investigación de los delitos y lucha contra el crimen, incluido el terrorismo ${ }^{55}$. Se establecieron garantías en lo que respecta al establecimiento de claras limitaciones en su uso, la prohibición de cesión a terceros países sin consentimiento expreso, límites en el periodo de retención de tales datos, derecho de acceso y rectificación, comunicación en el caso de que se produzcan quiebras a la seguridad y mecanismos de garantía jurisdiccional de los derechos vulnerados.

También debido a una decisión del Tribunal de Luxemburgo en el caso Schrems $(2015)^{56}$ se declaró ilegal el marco que regulaba la transmisión de datos en el área comercial entre EEUU y la UE, el conocido como «Safe Harbour Agreeement». Ello condujo a la negociación de nuevas condiciones que se recogieron finalmente en el «UU-US Privacy Shield», de febrero de 2016.

Con los nuevos Acuerdos, la UE, entre otras cosas, quiere blindarse ante el flujo de información masivo que se intercambia con otras potencias, como Estados Unidos,

53 TJUE Caso $c$-293/12 Digital Rights Ireland, 8 abril 2014.

${ }^{54}$ Decisión (UE) 2016/2220 del Consejo de 2 de diciembre de 2016 relativa a la celebración, en nombre de la UE, del Acuerdo entre los Estados Unidos de América y la UE sobre la protección de datos personales en relación con la prevención, la investigación, la detección y el enjuiciamiento de infracciones penales. El Acuerdo entró en vigor el 1 de febrero de 2017.

55 Su objetivo es proporcionar garantías adicionales a otros acuerdos de transferencia de datos ya anteriormente existentes.

56 TJUE Caso C-362/14 Maximillian Schrems v. Data Protection Commissioner. Tras las revelaciones de Snowden, el ciudadano austrico denunció a varias compañías como Facebook, Microsoft, Skype, Apple o Yahoo por lo que se derivaba de esas revelaciones, que habían estado cediendo los datos de sus usarios a la Agencia de Seguridad Nacional de Estados Unidos (NSA). El TJUE tuvo que analizar si el Acuerdo Safe-Harbour, en el marco del cual se permitía ese intercambio de información, era acorde con la normativa de la UE. En su sentencia el Alto Tribunal consideró lo siguiente:

Acerca de las leyes de los Estados Unidos;

1. «Normativa que permite a las autoridades públicas acceder de forma generalizada al contenido de las comunicaciones electrónicas lesiona el contenido esencial del derecho fundamental al respeto de la vida privada».

2. «Normativa que no prevé posibilidad alguna de que el justiciable ejerza acciones en Derecho para acceder a los datos personales que le conciernen o para obtener su rectificación o supresión no respeta el contenido esencial del derecho fundamental a la tutela judicial efectiva».

Explicó también en la sentencia;

3. «la protección del derecho fundamental al respeto de la vida privada al nivel de la Unión exige que las excepciones a la protección de los datos personales y las limitaciones de esa protección no excedan de lo estrictamente necesario»

y que por lo contrario las leyes americanas;

«autorizan de forma generalizada la conservación de la totalidad de los datos personales de todas las personas cuyos datos se hayan transferido desde la Unión a Estados Unidos, sin establecer ninguna diferenciación, limitación o excepción en función del objetivo perseguido y sin prever ningún criterio objetivo que permita circunscribir el acceso de las autoridades públicas a los datos y su utilización posterior a fines específicos». 
especialmente desde el caso de espionaje por parte de los servicios de inteligencia estadounidenses (la NSA). En principio, y siguiendo la jurisprudencia del TJUE, lo que la UE quiere evitar es que los datos de sus ciudadanos puedan ser objeto de daños a su privacidad sin que exista una razón legítima que lo justifique. La lucha contra el terrorismo yihadista es una causa que podría justificar ese intercambio de información entre Estados por la utilidad que esa inteligencia puede ofrecer en la persecución y prevención de nuevos ataques, incluida información sobre viajeros. Pero esos intercambios de datos deben producirse cuando se persiga un objetivo concreto y sean necesarios. Para ello la actuación debe asegurar todas las garantías legalmente establecidas y ha de ser proporcional.

En todo caso, especialmente cuando se trata de ceder datos en el marco de la lucha antiyihadista, la visión estadounidense y europea es diferente en ese balance entre privacidad y seguridad. La política estadounidense ha optado por una apuesta por la seguridad mucho mayor que la que derivaría del equilibrio que propone el Tribunal de Luxemburgo. De hecho, el refuerzo de esa seguridad que el Presidente Trump está abanderando puede poner en riesgo la efectividad de los Acuerdos que hemos mencionado.

\section{EL USO EN LAS FRONTERAS DE PERFILES Y LISTAS NEGRAS DE PRESUNTOS TERRORISTAS}

Lo cierto es que toda la información a la que nos hemos estado refiriendo más arriba, tras su debido tratamiento, puede ayudar a crear lo que se llaman perfiles de presuntos terroristas o sospechosos delincuentes. La creación de listas para monitorear y controlar sus movimientos, para establecer controles especialmente restrictivos con los nacionales de determinados países en las fronteras o simplemente impedir la entrada en un país por su vinculación con el terrorismo, o por ser nacional de determinados Estados constituyen un fenómeno conocido ya hace muchos años. Suponen un ejemplo más de cómo ello puede afectar a la libertad de movimiento de los individuos, sin entrar ahora en si tal medida puede considerarse justificada en esa búsqueda de la seguridad global que se pretende.

Así, desde los ataques a las torres gemelas estas listas se engrosaron ostensiblemente. El Consejo de Seguridad de Naciones Unidas fue el primero en elaborar las conocidas como «listas negras» donde se incluían los nombres y datos de individuos relacionados, de un modo u otro, con actividades terroristas y se decretaban determinadas medidas restrictivas contra ellos. La inclusión de una persona en una de estas listas negras puede suponer no solo el sometimiento a un seguimiento y escrutinio de sus movimientos, sino también la congelación de fondos u otras sanciones económicas, y puede conducir a la prohibición de entrada en un país o la denegación del reconocimiento del derecho de asilo.

Precisamente la transposición por la UE de las restricciones/sanciones a las que obliga Naciones Unidas en el asunto Kadi puso sobre la mesa la importante cuestión 
de la interrelación entre el cumplimiento de los compromisos internacionales y el respeto de los Derechos humanos en la Unión.

Kadi fue identificado como un posible defensor de al-Qaida por el Consejo de Seguridad de la ONU, se le incluyó en una lista de terroristas y fue sancionado. La UE transpuso esa sanción de la ONU a través del citado Reglamento 188/2002, que imponía medidas restrictivas contra personas y entidades vinculadas a Bin Laden, la red al-Qaida y los talibanes, incluidas en una lista adjunta. El Reglamento fue impugnado por Kadi ante los tribunales de la UE. Kadi alegó que no había sido informado de los motivos de su inclusión en la lista de personas y entidades sujetas a las sanciones establecidas. Por lo tanto, no había tenido la posibilidad de presentar un recurso judicial contra dichos motivos y, en consecuencia, su derecho a ser oído, así como su derecho a la tutela judicial efectiva, habían sido vulnerados. El Tribunal de Justicia ${ }^{57}$ examinó la legalidad de la normativa de la UE, que trasponía la Resolución de la ONU, con el argumento de que la protección de los derechos fundamentales forma parte de los fundamentos del orden jurídico de la UE. De acuerdo con este principio, todas las medidas de la UE deben ser compatibles con los derechos fundamentales, de lo que resultaba la aplicación preferente del Derecho de la Unión frente a la ejecución de compromisos internacionales.

En relación a esa inclusión de personas en las listas de terroristas, el TJUE ha añadido que «los nombres de personas, grupos o entidades se pueden mantener en la lista solo si el Consejo revisa periódicamente su situación. Todos estos asuntos deben estar abiertos a la revisión judicial» ${ }^{58}$.

La cuestión que se planteó en el caso Kadi y en los siguientes sigue estando vigente y es extrapolable a otras esferas. Así, por ejemplo, cabe preguntarse si, ante las respuestas globales o internacionales que puedan adoptarse en la lucha contra el terrorismo, los Estados miembros de la UE pueden y deben verificar la ejecución de tales medidas de conformidad con sus normas Constitucionales y también con los Tratados supranacionales que exigen el respeto de los derechos fundamentales. Por supuesto, la respuesta debe ser afirmativa por el principio de favor libertatis, debiendo optarse siempre por la interpretación que ofrezca mayores garantías a los derechos.

Lo cierto es que la posibilidad de no permitir la entrada de un individuo en un país, negándole el asilo por vínculos con el terrorismo está contemplada en la normativa internacional y nacional de asilo. De hecho, era admitida recientemente por el Tribunal de Justicia de la UE en el asunto Commissaire général aux réfugiés y los apatridas y Mostafa Lounani, de 31 de enero de $2017^{59}$, un supuesto de denegación de asilo

57 Sentencia del Tribunal de Justicia (Gran Sala) de 3 de septiembre de 2008. Caso Yassin Abdullah Kadi y Al Barakaat International Foundation contra Consejo de la UE y Comisión de las Comunidades Europeas.

58 El caso Kadi fue la base para nuevas decisiones como las adoptadas en el asunto Kadi II, y en otros como el asunto Omar Mohammed Othham de 11 de junio de 2009, el caso Al-Aqsa, de 15 de noviembre de 2012, o el Abdulabsit Abdulrabim de 14 de enero de 2015.

59 Asunto C-573/14. 
en Bélgica porque el peticionario había participado en actividades de grupos terroristas en el pasado.

La recopilación de datos para realizar perfiles de posibles sospechosos terroristas ${ }^{60}$, puede ser muy útil como medida antiterrorista e incluso necesaria, pero ha de realizarse con las debidas garantías por las consecuencias que puede acarrear para los derechos de los individuos afectados. En primer lugar, acaba estigmatizando a determinados colectivos dentro de la sociedad, colectivos de individuos que incluso pueden tener la nacionalidad del país donde se realizan tales controles. Hay individuos que por la religión que profesan y lugares de culto a los que acuden, hábitos de comportamiento, o vestimenta, pueden entrar a formar parte de una lista o base de datos de permanentes sospechosos, o ven recrudecidos los controles de entrada o salida del país, o se encuentran sometidos a continuas identificaciones por los agentes de seguridad. Estas actuaciones encierran un componente discriminatorio evidente, porque señala a todo un colectivo, con poca atención al principio de inocencia y demasiada a los rasgos étnicos, religiosos u otros. De hecho, desde la oleada de ataques yihadistas las identificaciones por perfil étnico han aumentado significativamente. Puede ponerse como ejemplo más llamativo la decisión impulsada por Donald Trump de impedir la entrada en EE.UU. de todo nacional de determinados países de mayoría musulmana o de los países vecinos al territorio ocupado por el ISIS.

Y a ello cabe añadir, en segundo lugar, que la recolección de datos para la elaboración de perfiles de sujetos peligrosos puede dar lugar a falsos positivos, como se ha demostrado. Los falsos positivos refieren a situaciones en las cuales un individuo inocente es identificado como sospechoso basándose en una incorrecta identificación por su perfil. Valga como muestra la de Jean Chales de Menzes, 27 años, que murió tiroteado por la policía en el metro de Londres un día después del desmantelamiento de un atentado en el mismo metro, al ser visto por las cámaras de seguridad y seguido como sospechoso y verle entrar en otra estación de metro ${ }^{61}$. Por lo tanto, los agentes que conducen la investigación y que, necesariamente, tienen sus listas de datos y sospechosos a los que investigan, deben adoptar las medidas necesarias para no caer en esos errores. $\mathrm{Y}$ han de conducir su investigación y trabajar en la recopilación de toda esa información sobre elementos de sospecha suficientes y razonables, nunca por el exclusivo perfil étnico o religioso.

\section{A MODO DE CONCLUSIÓN}

El terrorismo yihadista está dejando un legado de dolor no solo en Europa, sino en otras partes del mundo. La lógica respuesta de los Estados, y entre ellos los euro-

${ }^{60}$ AMNISTÍA INTERNACIONAL (2004). Threath and humiliation: Racial profiling, Domestic Security and Human Rights in the United States. New York, Amnesty International USA Publications.

${ }_{61}$ BAKKER, Edwin (2015). Terrorism and Counterterrorism; Comparing Theory and Practice. LPU General, Leiden, pág. 148. 
peos, es la persecución de los perpetradores de tales ataques y fundamentalmente la adopción de medidas de prevención y defensa de la seguridad nacional. Las respuestas que se han dado han sido de naturaleza variada, muchas de ellas consideradas desmesuradas, excesivas o posiblemente infructuosas. En la gran mayoría de las ocasiones han supuesto una clara restricción de los derechos fundamentales, con un desequilibrio no deseado entre seguridad y libertades. Entre esa variedad de medidas adoptadas en defensa de la seguridad nacional frente al terrorismo se ha producido el recrudecimiento de los requisitos de entrada en las fronteras y los controles en los aeropuertos y otros puntos de entrada, que se han vuelto especialmente incisivos, e incluso en ocasiones discriminatorios para individuos procedentes de determinadas partes del mundo.

Como se ha puesto de manifiesto en este trabajo, el intercambio de datos sobre el tránsito de individuos por las fronteras constituye una herramienta de extrema utilidad en esa lucha por garantizar la seguridad frente al terrorismo. La importancia del intercambio de datos respecto de pasos fronterizos y Costas volvió a ser puesto de manifiesto por el Consejo de la UE en junio de $2017^{62}$. Sin embargo, hay principios que deben respetarse.

Lo cierto es que la combinación de dos fenómenos distintos, migración y terrorismo, está poniendo a prueba uno de los símbolos más preciados de la integración europea: la libre circulación en el espacio Schengen. Incluso, como decíamos más arriba, se produce una confusión buscada entre inmigración y terrorismo. De hecho, se ha justificado el recrudecimiento de la normativa migratoria en la necesidad de frenar el terrorismo en Europa, en que se quiere prevenir la entrada de posibles terroristas yihadistas. Es algo así, tan absolutamente desmesurado, como decir: «vigilad las fronteras, que viene los terroristas». Nos encontramos ante fenómenos globales que, como se ha comprobado, exigen respuestas supranacionales y coordinadas. Pero no pueden ser abordados del mismo modo. Quizá en esto estemos empezando a adoptar una visión diferente. Así, en el Plan de Estrategia Nacional española presentado en 2013, se hablaba de riesgos y amenazas para nuestra seguridad nacional, enumerándolas e incluyendo el terrorismo y los flujos migratorios como iguales en el mismo listado. En esto se ha dado un paso, pues en el nuevo plan de estrategia nacional de diciembre de $2017^{63}$, el terrorismo es considerado un riesgo para la seguridad nacional, mientras los flujos migratorios son considerados «desafíos que, como retos y sin tener la entidad de amenaza, suscitan vulnerabilidad, provocan situaciones de inestabilidad o pueden propiciar el surgimiento de nuevas amenazas» (pág. 12). Efectivamente, parece difícil entender los flujos migratorios como amenazas, se trata más bien de un reto y, ni siquiera parece fácil pensar que pueden propiciar el nacimiento de nuevas amenazas.

Igualmente hay que recordar que hay determinados principios que deben regir esas políticas antiterroristas y, en concreto, los que regulan el tratamiento de datos

${ }^{62}$ Council Conclusions on EU External Action on Counter-terrorism (19 June 2017) (Ref. 10384/17)

63 Plan de Estrategia Nacional 2017, aprobado por el Consejo de Ministros el 1 de diciembre de 2017. 
que se llevan a cabo en el flujo de entrada en las fronteras. Así, la Asamblea General de Naciones Unidas ha subrayado en más de 60 resoluciones relacionadas con el terrorismo internacional la importancia fundamental de garantizar el respeto de los derechos humanos en la lucha contra el terrorismo. Y en concreto, en la Resolución aprobada por la Asamblea General, de 18 de diciembre de 2007, de protección de los derechos humanos y libertades fundamentales en la lucha contra el terrorismo, ya se exhortó a los Estados «a que se aseguren de que en todas las operaciones de control de fronteras, al igual que en los mecanismos previos a la entrada, se sigan directrices y prácticas claras y se respeten plenamente las obligaciones que tienen de conformidad con el derecho internacional, en particular el derecho de los refugiados y las normas de derechos humanos, respecto de quienes soliciten protección internacional»

Los tratados supranacionales también exigen el respeto de los derechos fundamentales en ese momento del cruce fronterizo. Y las normas aprobadas en materia de tratamiento de datos imponen reglas imperativas en cuanto a la protección de los mismos. El Tribunal de Justicia de la UE los ha subrayado con detenimiento como hemos podido ver en este trabajo ${ }^{64}$.

Por último, no cabe olvidar el principio de favor libertatis, debiendo optarse siempre por la interpretación que ofrezca mayores garantías a los derechos. De conformidad con la jurisprudencia del Tribunal de Luxemburgo, toda excepción al principio fundamental de libre circulación de las personas debe interpretarse de modo estricto. Por lo que hemos de entender que cualquier limitación a la entrada, en muchas ocasiones sobre la base de la peligrosidad que se desprenden de los datos de un individuo, debe efectuarse solo si presupone la existencia de una amenaza real, actual y suficientemente grave ${ }^{65}$, que afecte a un interés fundamental de la sociedad.

La cuestión que late en el fondo es siempre la misma: cómo lograr un equilibrio entre seguridad y libertad. El problema es que cada vez que se produce un nuevo atentado se desencadena una necesidad mayor de garantizar la seguridad y los ciudadanos parecen estar más dispuestos a sacrificar su esfera de libertad y la libertad de los otros.

${ }^{64}$ Conviene recordar de nuevo lo indicado en el caso C-362/14 Maximillian Schrems v. Data Protection Commissioner, o en Digital Rights Ireland c-293/12.

65 Tal vez los nuevos pasos de la política de la Unión puedan ir por ahí. Así, en enero de 2017, la Comisión, junto con los Estados miembros y el Servicio Europeo de Acción Exterior, inició los trabajos para crear una matriz que combine la evaluación del riesgo y elementos detallados de vulnerabilidad, a fin de sentar las bases para la fijación de prioridades y la coordinación de los esfuerzos de capacitación externa. 
Title:

Controlling data on EU cross-border movements as a mechanism to safeguard national security

\section{Summary:}

1. Jihadist terrorism as a cross-border phenomenon. 2. The benefit of data exchange on crossing-borders in the Schengen area. 3. New guidelines on data processing and the safeguard of national security. 4 . The register of passengers (The Personal Name Record or PNR). 5. When the data cross the external borders. The exchange of data with third countries. 5.1. The failed PNR Agreement with Canada and the EU Court of Justice's standards regarding the transfer of passengers' data. 5.2. The exchange of data with the United States. The EU-US Umbrella Agreement and the Privacy Shield. 6. The use of profiles and blacklists of alleged terrorists in cross-bording. 7. Conclusions

\section{Resumen:}

La gestión coordinada de las fronteras y el funcionamiento eficaz de los sistemas de tratamiento de datos de circulación de personas pueden servir como mecanismo de alerta temprana frente al riesgo de ataques terroristas. Puede fortalecer la capacidad colectiva de los Estados para detectar, prevenir y combatir el terrorismo al facilitar el intercambio oportuno de información, permitiendo así adoptar de forma responsable decisiones cruciales.

Este trabajo analiza los concretos instrumentos de gestión de datos en fronteras que pueden ser útiles en la lucha antiterrorista, porque el primer paso en inteligencia reside en la obtención de información, que luego será analizada y tratada para convertir esa información en conocimiento. Como tendremos oportunidad de comprobar, muchas de las bases de datos en fronteras se crearon para controlar la entrada de inmigrantes en las fronteras europeas, pero la información que ofrecen dichos sistemas puede servir también para luchar contra ese reto que nos amenaza, el del terrorismo yihadista. No obstante, este trabajo subraya que se trata de fenómenos distintos. Es cierto que la nueva oleada de ataques yihadistas ha coincidido, en el mismo espacio temporal, con la mayor crisis migratoria a la que se ha tenido que enfrentar Europa debido a crisis humanitarias y posteriormente a la guerra de Siria u otros conflictos. Pero, no son lo mismo. El terrorismo yihadista y la inmigración poco o nada tienen que ver, por mucho que se hayan querido vincular o se hayan pretendido justificar determinadas políticas contra la inmigración como algo necesario para luchar contra el terrorismo yihadista, con el fácil argumento de que frenando la inmigración se evita la entrada de potenciales terroristas en Europa.

El trabajo advierte del riesgo de que la lucha contra el terrorismo sea utilizada para reforzar los controles de personas en las fronteras con el verdadero objetivo de frenar los flujos migratorios. Al tiempo, subraya la 
necesidad de que en dichos controles se sigan directrices y prácticas claras y se respeten plenamente las obligaciones que los Estados tienen de conformidad con el Derecho internacional, tal como ha recordado el Tribunal Europeo de Derechos Humanos y el Tribunal de Justicia de la Unión Europea. De hecho, no son pocos los casos en los que estos Tribunales han subrayado la relevancia indubitada de principios como la reserva de ley, la necesidad o la proporcionalidad como sustrato de la licitud de muchas medidas que incluyen el tratamiento de datos personales.

\section{Abstract:}

EU Coordinated border management and effective functioning of data processing systems related to the movement of persons may serve as an early warning mechanism against the risk of terrorist attacks. It can strengthen the collective capacity of States to detect, prevent and combat terrorism by facilitating the timely exchange of information, thereby enabling crucial decisions to be adopted in a responsible manner.

This paper analyzes the concrete border data management tools that can be useful in the fight against terrorism. The first step in intelligence lies in obtaining information, which will then be analyzed and treated to turn that information into useful knowledge. As we will have an opportunity to verify, numerous border databases were created to control the entry of immigrants into European borders, but the information offered by these systems can also serve to fight against this challenge that threatens us, that of jihadist terrorism.

Nevertheless, we emphasize that terrorism and immigration are different phenomena. The truth is that the new wave of Jihadist attacks took place along the largest migratory crisis that Europe faced due to different humanitarian crises and to the war in Syria and other conflicts. But they represent different realities. Jihadist terrorism and immigration have little or nothing in common. In spite of this, many wish to link both with a view to justify certain anti-immigration policies as necessary actions for coping with Jihadist terrorism. This has been done based on a simple narrative: holding back immigration prevents the entry of potential terrorists in Europe.

This paper shows that the risk that the fight against terrorism will be used as a basis to reinforce people controls at the borders, while the true objective of these measures is to curb migratory flows. At the same time, it underlines the need for clear guidelines and practices to be followed when implementing such controls. It also vindicates the need for States to observe their obligations laid down by international law, as recalled by the European Court of Human Rights and the EU Court of the Justice. In fact, in many cases, these jurisdictions highlighted the undoubted relevance of the statutory reserve principle, the principle of necessity or the principle of proportionality, as legal basis for the adoption of measures that include personal data processing. 
Palabras clave:

Tratamiento de datos, cruce de fronteras, terrorismo, seguridad nacional, circulación de personas, inmigración

\section{Key words:}

Data processing, border crossing, terrorism, national security, movement of persons, immigration 